\title{
LAVI: Laboratório de Ambientes Virtuais Interativos
}

\author{
Patricia C. de Souza, Cristiano Maciel, Eunice P. Nunes, Karen Figueiredo, Luciana C. F. Borges ${ }^{\dagger}$ \\ Instituto da Computação \\ Universidade Federal de Mato Grosso \\ Cuiabá-MT, Brasil \\ patriciacs@ufmt.br, crismac@gmail.com, eunice@ufmt.br, \{karen,luciana\}@ic.ufmt.br
}

\section{RESUMO}

O objetivo deste artigo é relatar brevemente o histórico do Laboratório de Ambientes Virtuais Interativos (LAVI), sua criação, equipe, produções e interesses de pesquisa.

\section{PALAVRAS-CHAVE}

Interação Humano-Computador, Jogos Sérios, Tecnologias Assistivas, Privacidade, Legado Digital, Inteligência Ambiente, Ambientes Virtuais Tridimensionais. Gamificação.

\section{Introdução}

O Laboratório de Ambientes Virtuais Interativos (LAVI) foi criado em 2006 por três pesquisadoras do hoje extinto Departamento de Computação da Universidade Federal de Mato Grosso (UFMT). Na época, as principais linhas de investigação estavam centradas em Interação Humano-Computador, Tecnologias Educacionais e Realidade Virtual.

Atualmente, o LAVI está vinculado ao Instituto de Computação (IC) da UFMT e obteve, nestes 13 anos, um crescimento em termos de dimensão de pessoal e de novas abordagens de pesquisa o que pode gerar, por conseguinte, um alcance maior nos resultados das pesquisas desenvolvidas.

Por outro lado, percebe-se também uma limitação no desenvolvimento de pesquisas, uma vez que o Instituto de Computação não oferta programa de pós-graduação stricto sensu na área e tampouco em outra instituição na região. Dessa forma, os principais recursos humanos (professores e estudantes) que atuam no LAVI são oriundos dos dois cursos de graduação ofertados pelo IC: Ciência da Computação e Sistemas de Informação. Ainda, ocasionalmente, estudantes do Curso de Especialização em Engenharia Web e Governo Eletrônico, da qual fazem parte diversos professores do LAVI, realizam pesquisas na área.

O grupo de pesquisa conta atualmente com 13 pesquisadores da UFMT, cinco pesquisadores colaboradores de outras instituições e mais de 20 estudantes de graduação e pós-graduação lato sensu, que atuam em sete linhas de pesquisa. Ele está cadastrado no Diretório dos Grupos de Pesquisa do Conselho Nacional de Desenvolvimento Científico e Tecnológico (CNPq), tendo seus integrantes listados em http://dgp.cnpq.br/dgp/espelhogrupo/6749672220822058

Permission to reproduce or distribute, in whole or in part, material extracted from this work, verbatim, adapted or remixed, as well as the creation or production from the content of such work, is granted without fee for non-commercial use, provided that the original work is properly credited.

IHC 2019 - TRILHA FÓRUM DOS GRUPOS DE PESQUISA, Outubro 21-25, 2019, Vitória, Brasil. In Anais Estendidos do XVIII Simpósio Brasileiro sobre Fatores Humanos em Sistemas Computacionais. Porto Alegre: SBC

(C) 2019 by the author(s), in accordance with the terms of the Creative Commons Attribution-NonCommercial 4.0 International Public License (CC BY-NC 4.0).

\section{Principais Projetos}

Os projetos de pesquisa concluídos e em andamento aqui relatados possuem a IHC como linha de pesquisa principal ou secundária.

\subsection{Projetos Concluídos (Mais Recentes)}

A. Promovendo a Inteligência Ambiente: metodologia para desenvolvimento e suporte a sistemas colaborativos ubíquos (20132015) [1]. B. Identificação, verificação e resolução de conflitos entre normas e valores em sistemas multi-agente (2013-2015). C. Diretrizes para o Estabelecimento da Privacidade em Sistemas Ubíquos (2015-2018) [10, 15, 16, 17, 18].

\subsection{Projetos em Andamento}

D. Jogos Sérios e Gamificação para o Ensino de Computação e Sistemas de Informação (2016-Atual) - Esse projeto tem como objetivo geral estudar, desenvolver e aplicar práticas de jogos, tais como jogos sérios e gamificação, para o ensino de conteúdo das áreas de Computação e Sistemas de Informação, a fim de engajar e motivar os alunos e impactar de forma positiva no processo de ensino-aprendizagem. A IHC está atravessada nas duas primeiras etapas do projeto: estudo da interação no design de jogos e desenvolvimento de técnicas de interação para os jogos e produtos gamificados produzidos.

E. Projeto de Sistemas face ao Pré-Gerenciamento do Legado Digital Pós-Morte (2016-2020) - O projeto visa investigar, à luz dos princípios técnicos, culturais e legais, o legado digital pósmorte e propor soluções para a engenharia de sistemas computacionais no que se refere ao espólio digital deixado pelos proprietários das contas que falecem, por meio das expectativas dos atuais usuários da Internet. Também, a pesquisa visa investigar como aplicações Web, tais quais os memoriais digitais, têm sido desenvolvidas e qual a sua aceitação pelos usuários. Fazem parte do projeto nove instituições, com alunos de iniciação científica, especialização, mestrado e/ou doutorado. Encabeçados por pesquisadores do projeto, o tema compõe os Grandes Desafios de IHC [3] e os Grandes Desafios de Sistemas de Informação [11], no Brasil.

F. Concepção e Avaliação de Ambientes Virtuais Tridimensionais (2016-2020) - propõe metodologias inovadoras para concepção e avaliação de Ambientes Virtuais 3D (AV 3D) seguindo duas vertentes: i) AV 3D como instrumento pedagógico e ii) AV 3D para fins terapêuticos. A metodologia de pesquisa inclui revisões sistemáticas na literatura; estudos de campo e interações com profissionais das áreas de educação e saúde que são o foco da 
pesquisa; desenvolvimento de protótipos e validação. A área de IHC está presente neste projeto em três frentes de trabalho: i) projeto de interface com base em metodologias de Design Participativo; ii) aspectos de acessibilidade; iii) interação 3D. Para validar as metodologias propostas, são construídos mundos virtuais 3D baseados em jogos para serem aplicados em situações reais de aprendizagem e fins terapêuticos. Os resultados têm beneficiado as áreas da Educação e Saúde, além de contribuir para as comunidades de IHC e Realidade Virtual, devido às metodologias propostas e validadas via pesquisa.

G. Desenvolvimento de tecnologias assistivas personalizadas na FATA (2010-2025) - Esse projeto visa definir e implantar uma estrutura de desenvolvimento de alta tecnologia assistiva que permite obter soluções personalizadas para pessoas com deficiência e, em reabilitação. Em uma Fábrica de Alta Tecnologia Assistiva (FATA) - promove-se um ambiente no qual profissionais da computação colaboram com profissionais de saúde e seus pacientes (pessoas com deficiência) no provimento de soluções computacionais personalizadas aos usuários finais, e, desta forma, atendem à diversidade das demandas desses pacientes.

H. Investigação de Novos Paradigmas que favoreçam o Entendimento e Decisões Assertivas Acerca dos Riscos de Privacidade e Segurança Digital (2018-2021) - visa investigar e realizar testes com modelos e técnicas das áreas de interação humano-computador e de segurança em sistemas e dispositivos móveis visando apontar problemas e propor soluções para minimizá-los de forma que possam fornecer aos usuários de tecnologias digitais maior entendimento sobre os riscos de privacidade e segurança ajudando-os a tomarem decisões mais assertivas em relação a proteção de seus dados pessoais.

\section{Soluções Desenvolvidas}

Nesta seção são descritas algumas das soluções desenvolvidas bem como modelos e/ou métodos de elicitação de requisitos, design participativo dentre outros. O LAVI também tem atuado junto a projetos em órgãos governamentais, como é o caso do Fundo Nacional de Desenvolvimento Educacional (FNDE) e o Tribunal de Contas do Estado de Mato Grosso (TCE-MT).

O projeto $B$ desenvolveu a ferramenta CSMA Modelling Tool para a modelagem de sistemas multi-agentes culturais no Eclipse com uma interface gráfica de modelagem.

O projeto $C$ desenvolveu um conjunto de diretrizes para apoiar projetistas de software no desenvolvimento de aplicativos em respeito ao Marco Civil e critérios de usabilidade [10] e user experience [15]. Também, desenvolveu um Método para Elicitação de Requisitos de Privacidade em Computação Ubíqua [16, 17] e o Método Mobile-PrivAccess [18].

O projeto D desenvolveu a plataforma chamada Game in Class [5], para a gamificação de cursos de computação e diversos jogos sobre conteúdo da área de computação, entre eles o Computasseia ${ }^{1}$ para o ensino de História da Computação.

O projeto $E$ tem trabalhado de forma inovadora na perspectiva de oferecer recomendações, protótipos e categorias conceituais para o projeto de legado digital. Ainda, tem realizado avaliação de soluções amplamente utilizadas no mercado, publicando-as no formato de artigos e relatórios técnicos. Também, tem atuado na concepção de um memorial digital dos doadores de corpos da Universidade Federal de Ciências da Saúde de Porto Alegre (UFCSPA). As produções deste projeto estão listadas em http://lavi.ic.ufmt.br/davi/pt/

\footnotetext{
${ }^{1}$ http://gameinclass.ic.ufmt.br/computassy
}

O projeto $F$ desenvolveu três serious games 3D disponibilizados na Play Store: 1) Hospital Mirim [7] - Brinquedo Terapêutico Digital Interativo com propósito de simular procedimentos médicos cotidianos como, por exemplo, punção venosa que simula coleta de sangue e aplicação de medicamento; 2) Protegendo a Terra [6] - aborda o tema "Reciclagem, Reutilização e Redução de lixo". O game foi desenvolvido a fim de encorajar as crianças na preservação do meio ambiente, como uma forma de reduzir os impactos ambientais causados pela falta de conscientização dos cidadãos em seus contextos diários; 3) ABCDiabetes [14] prepara o público infantil/juvenil diagnosticado com Diabetes Tipo 1, sobre cuidados com a saúde durante o tratamento como, por exemplo, a melhor escolha dos alimentos e sua relação com o nível de glicose no organismo. O game está embasado em minijogos, sendo disponibilizado, neste momento, o primeiro minijogo - "Seleção de Alimentos", que de uma forma lúdica apresenta o medidor de glicose como uma barra de pontuação no game, e quanto mais o jogador selecionar alimentos saudáveis, maior será sua pontuação no game.

No projeto $G$ foram desenvolvidos dois comunicadores personalizados, por meio de metodologias de Design Participativo, para duas pessoas com sequelas de paralisia cerebral (uma criança de 4 anos, um adulto de 46 anos) [8][9].

\section{Prêmios}

Com relação aos projetos do LAVI supracitados, cita-se a seguir alguns prêmios recebidos. O artigo do projeto $G$ [8] ganhou o best paper do IHC'12 e foi convidado para publicação em um periódico [9]. $\mathrm{O}$ artigo [2], parceiro do projeto $E$, ganhou o best paper do IHC'16 e foi convidado para publicação em um periódico [12]. Ainda, deste projeto, o artigo [13] foi o best paper dos artigos resumidos do IHC'17. O artigo [4], a respeito do jogo de história da computação do projeto $D$, foi selecionado entre os best paper do $24^{\circ}$ Workshop sobre Educação em Computação (WEI 2016), recebendo uma menção honrosa. O artigo [5], que apresenta uma plataforma de gamificação produzida pelo projeto $D$, foi selecionado para as finais do concurso Apps.Edu 2015 do IV Congresso Brasileiro de Informática na Educação.

\section{Organização de Eventos}

Durante sua trajetória, pesquisadores do LAVI foram responsáveis pela organização de alguns eventos científicos, relacionados a distintas áreas:

- 2012 - Simpósio Brasileiro sobre Fatores Humanos em Sistemas Computacionais (IHC'12);

- 2013 - Simpósio de Realidade Virtual e Aumentada (SVR 2013);

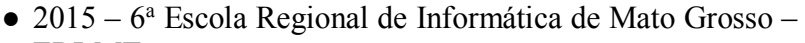
ERI-MT;

- 2015 - $1^{\text {a }}$ Jornada de Interação Humano-Computador para Empresas (Centro-Oeste);

- 2019 - XIII Workshop de Tecnologias da Informação e Comunicação das Instituições Federais de Ensino Superior no Brasil - WTICIFES. 


\section{Considerações Finais}

Com relação a integração entre os projetos e participantes destes, cabe salientar que os membros do LAVI compartilham infraestrutura física e há colaboração entre projetos, sempre considerando as especialidades e disponibilidades de cada um. Quando procurados para execução de parcerias com outros órgãos, o grupo aloca as demandas conforme exposto. No momento, os projetos atuam, em sua maioria, com alunos de iniciação científica.

Por estar no centro do país e ao mesmo tempo equidistante dos grandes centros de pesquisa, o LAVI necessita do apoio de outros grupos de pesquisas mais consolidados na área de IHC para alavancar suas pesquisas, bem como da criação de um programa de pós-graduação Stricto Sensu. O elo com empresas e órgãos governamentais tem sido outro anseio desse laboratório. Maiores informações podem ser adquiridas pelo site ou redes sociais do LAVI $^{2}$.

\section{AGRADECIMENTOS}

Agradecimentos ao apoio recebido pelas fontes de financiamento: Fapemat, Capes, CNPq; à Fundação UNISELVA e à UFMT, com suas várias unidades; a todos que colaboraram no crescimento do LAVI, em especial, aos estudantes; e, por fim, aos voluntários que participaram de atividades de apoio às pesquisas do grupo.

\section{REFERÊNCIAS}

[1] C. Maciel, P. C Souza, J. Viterbo Filho, F. F. Mendes and El. F Amal. 2015. A Multiagent Architecture to Support Ubiquitous Applications in Smart Environments Communications in Computer and Information Science, v. 498, 106-116.

[2] F. H. S. Pereira, R. O. Prates, C. Maciel and V.C. Pereira. 2016. Análise da Antecipação da Interação e de Aspectos Volitivos em Sistemas de Comunicação Digital Póstuma. In Proc. of the XV Simpósio sobre Fatores Humanos em Sistemas Computacionais (IHC 2016), São Paulo, SP.

[3] M.C.C. Baranauskas, C.S. de Souza, and R. Pereira. 2015. I GranDIHC-BR Grand Research Challenges for Human-Computer Interaction in Brazil. HumanComputer Interaction Special Committee (CEIHC) of the Brazilian Computer Society.

[4] J. C. O. Santos, and K. S. Figueiredo. 2016. Computasseia: Um Jogo Para O Ensino De História Da Computação. In $24^{\circ}$ WEI - Workshop sobre Educação em Computação, Porto Alegre. XXXVI Congresso da Sociedade Brasileira de Computação, 2026-2035.

[5] J. M. Ribeiro, K. da S. Figueiredo and C. Maciel .2015. Game in Class: Criando Disciplinas Gamificadas. In I Concurso Integrado de Desenvolvimento de Soluções de Tecnologia e Objetos de Aprendizagem para a Educação (Apps.Edu), Maceió. IV Congresso Brasileiro de Informática na Educação.

[6] E. P. S. Nunes, A. R. Luz, E. M. Lemos, C Maciel and L. C. L. F. Borges 2016 Mobile Serious Game Proposal for Environmental Awareness of Children. In Proc. of the Frontiers In Education - FIE, Erie, Pensilvânia.

[7] E. M. Lemos, A. R. Luz, C. H. A. Gontijo, D. A. C. Santos, C. Maciel, and E. P. S. Nunes. 2016. Hospital Mirim: um serious game como Brinquedo Terapêutico Digital Interativo no apoio a procedimentos médicos. In Proc. of the XV Simpósio Brasileiro de Jogos e Entretenimento Digital, São Paulo.

[8] L.C.L.F. Borges, L. Filgueiras, C. Maciel, and V.C. Pereira. 2012. Customizing a Communication Device for a Child with Cerebral Palsy using Participatory Design Practices: Contributions Towards the PD4CAT Method. In Proc. of the IHC 2012 - Simpósio de Fatores Humanos em Sistemas Computacionais, CuiabáMT.

[9] L.C.L.F. Borges, L.V. Filgueiras, C. Maciel, and V. Pereira. 2014. The life cycle of a customized communication device for a child with cerebral palsy: contributions toward the PD4CAT method. Journal of The Brazilian Computer Society (Online), v. 20, p. 10

[10] E.A. Yamauchi, P.C. Souza, and D. P. Silva Júnior. 2016. Questões Proeminentes para o Estabelecimento da Privacidade em Políticas de Privacidade de App Móveis. In Proc, of the XV Simpósio sobre Fatores Humanos em Sistemas Computacionais (IHC 2016), São Paulo, SP

[11] C. Maciel, V. C. Pereira. 2017. Technological and Human Challenges to Addressing Death in Information Systems. In: I GranDSI-BR - Grand Research Challenges in Information Systems in Brazil - 2016-2026.1 ed.: Brazilian Computer Society (SBC), 161-174.
[12] F. H. S. Pereira, R. O. Prates, C. Maciel, V. C. Pereira. 2017. Combining Configurable Interaction Anticipation Challenges and Volitional Aspects in the Analysis of Digital Posthumous Communication Systems. In: SBC Journal on Interactive Systems. v.8, n.2, $77-88$.

[13] V. F. Galvão, C. Maciel. 2017. The Acceptability of Digital Immortality: Today's Human is Tomorrow's Avatar In: XVI Simpósio Brasileiro sobre Fatores Humanos em Sistemas Computacionais (IHC 2017), Joinville, SC. In Proceedings of the 16th Brazilian Symposium on Human Factors in Computing Systems. Porto Alegre, RS: SBC, 2017. p.557 - 560 .

[14] G. A. Gutierrez, I. Jordany, D. A. C. Santos, G. Chireia, R.M.E.M. Costa, L.C.L.F Borges, E.P.S. Nunes. 2018. ABCDiabetes: um Jogo Sério 3D para Conhecer Melhores Práticas sobre o Tratamento de Diabetes". In Proc. II Workshop de Iniciação Científica - WIC-SVR 2018, Foz do Iguaçu, Brasil.

[15] D. P. Silva Junior, P. C. Souza, C. Maciel. 2016. Establishing Guidelines for User Quality of Experience in Ubiquitous Systems. Lecture Notes in Computer Science, v. 9749, 46-57.

[16] D. P. Silva Junior, P. C. Souza and C. Maciel, 2018. Privacy Requirements Elicitation in Ubiquitous Computing. In Proceedings of the 22th Brazilian Symposium on Software Engineering. ACM.

[17] D. P. Silva Junior, P. C. Souza and T. A. J Gonçalves. 2018. Early Privacy. In: the 17th Brazilian Symposium, Belém. Proceedings of the 17th Brazilian Symposium on Human Factors in Computing Systems - IHC 2018. New York: ACM.

[18] R. T. Chicanelli, L.C.L.F. Borges, P.C. Souza. 2018. Mobile-PrivAccess: Method for Analyzing Accessibility in Mobile Applications from the Privacy Viewpoint Abiding by W3C. Lecture Notes in Computer Science, v. 10907, 18-37.

${ }^{2}$ www.lavi.ic.ufmt.bre @laviufmt 